\title{
Stress and Eating Behaviour: Implications for Obesity
}

\author{
Clare Scott Alexandra M. Johnstone \\ University of Aberdeen Rowett Institute of Nutrition and Health, Aberdeen, UK
}

\section{Key Words}

Obesity $\cdot$ Workplace stress $\cdot$ Eating behaviour $\cdot$ Personality $\cdot$ Shift work

\begin{abstract}
Background: This report outlines our strategy to examine the influence of workplace stress on eating behaviour, discussing the current literature which explores the relationship between stress and eating behaviour. This research aims to add to and develop the current understanding of the links between stress and eating behaviour. Specifically the aims are to examine the effect of workplace stress in both day workers and shift workers and their subsequent eating behaviour. Methods: The effect of healthy working environment initiatives on stress and eating behaviour will also be examined by comparing a workplace with such an initiative to one with no such initiative. The role of personality on both eating behaviour and stress susceptibility will be examined. In order to achieve this, 450 individuals from 3 public sector workplaces will be recruited. Anthropometric measurements (waist-hip ratio, BMI, visceral fat percentage) will be assessed, as well as personality, eating behaviour profiles, food intake (7-day weighed intake food diary) and both individual daily stressors as well as workplace stress assessed using the demand/control model of job strain will be assessed. Conclusions: Implications for policy and future research are also discussed.

Copyright (C) 2012 S. Karger AG, Basel
\end{abstract}

\section{Introduction}

There is a well-documented rapid rise in obesity affecting Western culture that is linked to an increase in associated chronic health conditions [1]. Gaining a greater understanding of the underlying physiology and psychology influencing eating behaviour is therefore a current and topical research focus. There are many factors influencing feeding behaviour and appetite control [2]. However, individuals do not always initiate eating due to (learned) 
internal physiological symptoms of hunger; it is recognised that psychological and external (environmental) cues can promote overeating, at least in the short term [3-5]. Highly palatable food and snacks can contribute to overconsumption of calories, particularly through promotion of hedonic or reward mechanisms. Stress or 'daily hassles' has been shown to increase snacking behaviour, particularly for high-fat and high-sugar foods [5], which may contribute to our 'obesogenic environment'.

The relationship between stress and eating behaviour has been explored from several different domains. In psychology, the relationship between the two has been observed with eating behaviour identified as susceptible to change as an emotion coping strategy $[3,6]$. In animal studies, the relationship between stress and eating behaviour has been explored as part of a physiological and biological reaction and has, in particular, led to knowledge of the neuronal food reward and neuro-endocrine systems that influence eating behaviours [4]. Such research has resulted in a growing body of evidence supporting the premise that stress influences health in two ways; directly though autonomic and neuroendocrine responses and indirectly though changes to health behaviours $[7,8]$.

\section{Workplace Stress}

While it is recognised that the health of people who work is generally better than that of those that do not, it is also recognised that there are circumstances in which the burden of work may contribute to unhealthy behaviour(s) [9]. The workplace could be the origin of various health inequalities since working conditions are associated with employees' health behaviour. Unfortunately, stress is a feature of everyday modern life, particularly so in the workplace. A recent survey conducted by the UK Trade Union Congress estimated at $62 \%$ of workers experience feelings of stress in their workplace with 1 in 10 seeking support from their general practitioner for work-related stress [10]. It is increasingly recognised that the economic cost of workplace stress is significant. Within the UK, it is estimated to cost the economy nearly $10 \%$ of its Gross National Product. Aside from the financial impact, stress has been causally linked to many negative health outcomes, including heart disease, diabetes and obesity $[11,12]$.

The role of the employee and employer can influence stress levels, since 'demand' and 'control' both play important roles in perceived stress levels in the workforce [13]. Individuals who experience high demand and low control may be at greater risk of stress. However, the degree to which one is susceptible to stress also depends on the individual's personality phenotype.

There have been a few studies conducted within workplaces in order to assess the impact of specific work-related stress on eating behaviour. Wardle et al. [6] assessed the association between work stress and eating behaviour in 90 workers, and the results indicated that high work stress was associated with elevated energy, saturated-fat and sugar intake in comparison to individuals with low work stress. In this instance, however, high and low work stress was defined by hours worked, rather than specific work-induced stressors. O'Connor et al. [7] examined the effect of hassles and stressors in the workplace on eating behaviour in a large sample $(n=422)$ of workers from one organisation. Daily food diaries recorded food intake between meals, and each stressor experienced was also recorded along with a rating of how 'stressful' the particular event had been. Results revealed that increased daily hassles were associated with increased snacking on highenergy, high-fat sugar snacks. In particular the increased snacking occurred in response to ego-threatening, interpersonal and work-related hassles only. 
Research investigating workplace stress and eating behaviour has thus far primarily focused on day workers. However, compared to individuals who work during the day, shift workers have been identified to be at greater risk of metabolically related disorders. One in 5 workers in Europe is employed on shift work involving night work, and more than 1 in 20 have extended work hours [14]. Evidence indicates that, while total energy intake does not vary between day and shift workers, the type of food, typically high-energy, easy-to-prepare food, and energy distribution throughout the day does vary. While it is the case that there are other variables which may account for the differences in eating behaviour between day and shift workers, such as circadian disruption and sleep deprivation, it may also be the case that shift workers are more susceptible to perceived stress. The need for greater exploration of the relationship between shift workers and their diets has been highlighted in previous research [15]. Since many adults spend a substantial amount of their time at work and consume at least one meal at work each day, a healthy workplace environment is important, particularly as jobs become more sedentary. An increased prevalence of obesity among employees can have indirect economic consequences for employers, in terms of productivity through illness and absenteeism of employees. Research that examines the relationship between stress and eating patterns and how employer healthy lifestyle provision impacts on behaviours is lacking.

\section{Psychological Evidence for the Relationship between Stress and Eating Behaviour}

There is evidence to suggest that the majority of people tend to change their eating behaviour when they believe themselves to be stressed, with an estimated $80 \%$ of people altering their caloric intake either by increasing or decreasing their consumption [4]. The direction of change of calorie intake is highly dependant on eating behaviour profile and personality phenotype. For example, it is well documented that individuals who are 'restrained' in their eating behaviour are more susceptible to stress-induced eating and have been shown to be more susceptible to the deleterious effects of stress on eating behaviour (overeating) $[8,16]$. Dietary restraint refers to the tendency to restrict food intake in order to control body weight. Dietary restraint can also be associated with 'disinhibition' and loss of control over eating resulting in binge eating. Zellner et al. [8] reported that $71 \%$ of those woman who reported increasing their food intake when stressed were categorised as restrained eaters compared to only 35\% of those who did not alter their eating behaviour, or reduced their food intake, in response to stress. Zellner's study was based on a self-report questionnaire of eating behaviour when stressed and, as such, it is not possible to conclude what manner of stressor people were considering when responding. Wallis and Hetherington [16] also found evidence that highly restrained individuals increased their snacking after ego-threatening stressors compared to unrestrained individuals.

Both chronic stress and acute stressors are believed to impact on eating behaviour [7, $17,18]$. The resulting changes to eating behaviour such as high fat intake and low fibre and fruit and vegetable intake may indirectly contribute to elevated cardiovascular disease and cancer risk. High levels of stress in individuals have also been found to be associated with increased saturated fat consumption and decreased overall calorie intake [6]. Thus, several phenotypes exist in relation to stress and eating. There is evidence suggesting that the impact of stress depends on both i) the type of stressor and ii) individual physiological and psychological differences. Evidence suggests that only stressors perceived as ego-threatening rather than 'physically threatening' result in altered eating behaviour [7, 16]. Egothreatening stressors can be characterised as a situation in which there is 'a fear of failure' 
with a potentially negative evaluation [7]. Interpersonal stress has also been implicated in eating behaviour; examples of forms of interpersonal stress include ostracism and argument [19]. Both ego-threatening and interpersonal stress appear to exert influence on individuals with existing psychological characteristics, such as restrained eaters $[7,19,20]$.

Stressful events are associated with a variety of behavioural responses, and generally people utilise different coping strategies in order to deal with the stressor. Emotional eating could serve as a coping mechanism for many individuals. Emotional eaters are regarded to be more likely to overeat under both general and specific stress than non-emotional eaters. Among emotional eaters, food cravings and consumption of food high in carbohydrates and fats increase in response to stressors [3]. Self-report measures of perceived stress and questionnaire-based assessment of eating behaviour revealed that emotional eaters were more likely to report overeating than non-emotional eaters who more likely to report undereating [16]. Specifically, the foods that were reported to be overconsumed were snack foods, including crisps, chocolate and biscuits, in response to specific stressors.

Other personality factors such as perfectionism and conscientiousness may also play a mediating role in stress and eating behaviour. Perfectionism has been associated with eating behaviours in both the general non-clinical population [21] and individuals with disordered eating [22]. Perfectionism is a personality characteristic that has also been associated with increased stress and maladaptive coping. While the role of perfectionism, stress and eating behaviour has been assessed previously, the focus has been on disordered eating. Such studies have demonstrated that stress situations reveal an association between perfectionism and unhealthy eating attitudes [23]. In this study, 42 female participants completed questionnaires on stress, perfectionism and eating attitudes and were assessed immediately following a stress-inducing task and again on an average day. However, further exploration of the influence of perfectionism on stress and eating style (restrained and emotional) and eating behaviour in the general population requires further investigation. Conscientiousness is 1 of the 5 major dimensions of personality and has been demonstrated to play a role in eating behaviour. Previously it has been linked to the Eating Disorder Inventory in clinical and non-clinical samples [24], and in 2001 it was suggested to play an important role in understanding eating behaviour [25]. The relationship between conscientiousness, perfectionism and stress has been demonstrated in a study of 155 participants who were assessed on all three of these traits as well as self-reported snacking levels. The results revealed that individuals who had low conscientiousness, but were highly self-oriented in perfectionism reported an increase in meal snacking during stressful periods [26]. Such studies demonstrate that stress can modify eating behaviours negatively, and such effects are likely to be as a result of a complex interaction of many individual factors.

Food intake can have a remarkable effect on mood and most individuals will have their own food-associated, mood-related habits e.g. a cup of coffee to get going in the morning or eating sweet foods to reduce anxiety. Emotional states can have major effects on eating behaviour and result in either overeating or undereating. There have been several reviews of studies concerning emotional eating in relation to body weight (e.g. Allison and Heshka, 1993 [27]). These studies have almostalways dealt with negative emotions such as depression or fear, comparing obese and normal-weight subjects, with results indicating relative overeating in obese individuals during negative emotional states. A psychosomatic interpretation has been that eating by obese individuals in response to negative emotions is a learned behaviour to reduce the negative state [28]. Geliebter and Aversa [29] conducted a questionnaire study of underweight, normal-weight and obese subjects and also similarly reported that the overweight group ate more than the other weight groups when experiencing negative emotions and situations, whereas underweight individuals reported eating more when experiencing positive emotions and situations. Most striking was the undereating by 
underweight individuals during negative emotions and situations, which may contribute to their being underweight. The relationship between mood and food will continue to be hotly debated at an individual and population level.

The phenomena of 'food craving' and 'food addiction' are not well researched, and indeed many researchers would question their existence. A food craving is 'an irresistible urge to consume a specific food', in particular linked to high-sugar and high-fat foods [30]. This area merits further investigation, since the link between eating behaviour(s) and food craving or addiction as a risk factor for obesity is not well understood. The neuropsychology of food reward and food choice and the links between the appetite regulatory network, eating behaviour and food preference are not understood. Future research will likely focus on brain neuroanatomy and interactions with food to challenge whether food is indeed 'addictive' and to investigate the pathways involved in the 'rewarding' aspects of food and whether this experience can be replicated in healthier food product alternatives, to control body weight.

\section{Physiological Evidence for the Relationship between Stress and Eating Behaviour}

Whilst the influence of psychology in eating behaviour is important, it is not the only influence by any means. Physiology also plays an important and influential role in individuals' eating behaviour. The physiological influence which stress has on neural networks bias an individual's ability to apply cognitions to behaviour. The secretion of hormones and activation of networks can result in an emotional activity and degrades executive control [4]. Dallman and colleagues [17] had previously examined the link between stress and the resulting physiological effect that this has on eating behaviour, in particular highly caloric food choices. Dallman et al. [17] established that in rodents that were chronically stressed the central corticotrophin-releasing factor (CRF) networks are recruited. Such CRF recruitment is associated with arousal as well as behavioural, autonomic and neuroendocrine changes that accompany the chronic stressor. However what was also demonstrated to occur was an elevation in glucocorticoids (GCs) which aim to counteract the effects of the central CRF and associated changes. The GCs have been demonstrated to stimulate a drive for the ingestion of 'comfort foods'. Dallman et al. [17] suggested that this consumption of comfort foods may negate the effects of the chronic stressor by reducing the HPA responses to the CRF responses in the hypothalamus. In these terms, the ingestion of highly caloric 'comfort foods' could be regarded as a self-medication approach to dealing with stress.

A recent study has also suggested that emotional eaters, who are using food as either an avoidant or emotion-focused response to a stressor may be influenced by hormonal responses that do not occur to the same extent in non-emotional eaters [3]. This study conducted on 48 women of either a high or low emotional eating status revealed a more pronounced elevation of cortisol levels in emotional eaters than in non-emotional eaters following a laboratory-based stress test. A further interesting finding from this study revealed that emotional eaters had a baseline ghrelin level higher than non-emotional eaters, and whilst the ghrelin levels declined in non-emotional eaters after food was given this was not the case in emotional eaters. The authors conclude that this lack of decline may explain why emotional eaters continue to sustain their eating behaviour.

The influence of cortisol reactivity in response to stress has increasingly been linked to eating behaviour, specifically consumption of highly caloric foods, with individuals who are high cortisol reactors to stress consumed more high-fat, sweet foods during the recovery period than those who were lower cortisol reactors. Yet much of the basis for such an observation lies in laboratory-based work, with no examination as to whether the predisposition 
to consume such foods remains in everyday situations. Such a paradigm was investigated by Newman et al. [5], who assessed cortisol reactivity in 50 women following a laboratorybased stressor. What was of particular interest in this study was that the subjects were then required to keep a 14-day food diary indicating snacks consumed as well as daily hassles (an indicator of daily stressors). Results revealed that increased daily hassles were associated with increased consumption of energy-dense snacks, yet this was only observable in the high cortisol reactors. This study in free-living subjects indicates that cortisol reactivity may pre-dispose individuals to stress-induced food intake.

Whether psychological and physiological in its basis, the influence of stress on eating behaviour is an established one and is a phenomenon which has significant implications for the health of European nations. Obesity is at epidemic levels in some regions and is a burgeoning problem for many western countries. Consequently, elucidating the underlying issues influencing such eating behaviour must be prioritised.

\section{Evidence Gaps in the Literature}

Despite the well-researched areas that have been discussed thus far, there remain gaps in the literature, and areas for future research. The first major limitations of the majority of studies investigating the link between stress and eating behaviour have been either their conduct in a laboratory setting or, where free-living subjects have been studied, questionnaires or free recall have been employed to ascertain eating behaviour and patterns $[6,16]$. The use of the gold standard assessment of food intake, 7-day weighed intake food diaries, in such research is lacking. In order to accurately assess eating patterns and dietary intake in relation to stress, future research must utilise the gold standard means of assessment.

While many studies have focused on improving eating behaviour through a workplace setting, the majority have done so from a dietary perspective and have shown that individualised interventions can impact on behaviour in the context of a workplace environment which, in turn, can positively impact on health. Few have assessed whether global healthy workplace environment initiatives also improve eating behaviour patterns compared to those workplaces that do not have such an initiative in place. As there is an increase in such initiatives, indeed many government-driven, this requires greater investigation. Shaping the work environment to reduce the incidence of disadvantageous eating patterns and promote healthier physical activity patterns in order to control calorie intake and body weight requires evidence-based research. In particular, research should focus on elucidating how typical stress situations in 'modern life' trigger disadvantageous eating patterns and preferences for certain foods.

Perhaps crucially given the rapidly increasing prevalence of workplace stress, relatively few studies have focused purely on the effects of workplace stress on eating behaviour. A keynote report commissioned for the Health and Safety Executive in 2005 focused on a sample of 17,000 workers in order to establish the scale of workplace stress and possible determinants of such stress [31]. This report established that it is possible to elucidate workplace stress from general life stress and furthermore have demonstrated that it is possible to examine the particular determinants of such stressors. Yet few studies have examined the effects of such stressors on eating behaviour and those that have tended to focus on day workers, usually within private organisations, where stress is generally less of a problem than in the public sector [32].

Lowden et al. [15] established that, compared to individuals who work during the day, shift workers are at higher risk of obesity, cardiovascular disease and a range of other metabolic diseases. They established that some, but not all, of the range of conditions were asso- 
ciated with poorer diet and disordered eating patterns, perhaps as a result of stress and physical inactivity as well as disrupted circadian rhythms. There is a need for greater exploration of the relationship between shift workers and their diets, in particular public workers (not just offshore and medical staff, where much of the published research has focused). Therefore the need for further examination of public sector workers and the inclusion of shift workers is certainly warranted.

\section{Future Research: NEUROFAST - the Effect of Workplace Stress on Eating Behaviour}

Workplace stress presents a rapidly growing burden on economies, which is co-occurring with the burden of the growing obesity epidemic and obesity-related health conditions. Stress is known to adversely affect eating behaviour in up to $80 \%$ of people, and accordingly a greater understanding of the underlying workplace stressors and how such stressors negatively interact with personality, eating patterns and actual eating behaviour is of value. Accordingly there is an identified need to further investigate the relationship between stress and eating behaviour in the workplace and its implications for obesity.

This EU-funded research programme aims to specifically examine the relationship between workplace stress and eating behaviour in greater depth in order to increase our understanding of stress-induced eating and its potential impact on body weight regulation. This research will help elucidate how typical stress situations in 'modern life' trigger disadvantageous eating patterns and preference for certain foods. Public sector workplaces have been identified as reporting greater instances of workplace stress than the private sector and as such will be the focus of this research programme. Due to the increase in healthy environment initiatives implemented within public sector organisations, this research also aims to examine whether environment can be shaped to influence healthier food choices. This will be achieved by examining workplaces with Scottish Government approved healthy environments compared to similar workplaces without this status. The need for greater examination of the influence of shift work on stress and eating behaviour has received relatively little research previously and so will also be assessed within this research. Lowden et al. [15] indicated that more research is required in this group of workers who are known to be at greater health risk than non-shift workers. This research will take place in freeliving subjects, using the gold standard 7-day weighed intake food diary in order to ascertain eating patterns, including snacking. Hourly rating of stress, as well as detailed recordings of daily stressors will also be assessed.

What research will we carry out on this topic?

We will assess the impact of workplace stress on eating behaviour across three public sector organisations. One such workplace will encompass shift workers.

We will assess the impact of workplace shift patterns on daily stress and eating behaviour in a large group of men and women.

We will assess the impact of a health-promoting work environment on daily stress and eating behaviour in a large group of men and women.

We will assess how personality profile impacts on eating patterns and stress - as facets of the 'big five', neuroticism, extroversion, openness, agreeableness \& conscientiousness as well as the additional facet of perfectionism have all been demonstrated to be associated with stress susceptibility as well as eating behaviour profiles, all these aspects will be assessed. It is believed that there are likely to be 'stress-susceptible' and 'stress-resistant' phenotypes who do or do not alter food intake in times of stress. 
Both eating behaviour profile, whether individuals are restrained, disinhibited, emotional or external eaters, will be examined, however importantly actual food intake will be assessed by way of a 7-day weighed intake food diary.

Stress itself will be assessed both by recording of daily hassles that the individual perceives as stressful, hourly VAS ratings of perceived stress and an assessment of job strain and stress using the Karasek categorisation of Demand, Control, Effort and Reward [13].

We will inform policy makers and policy implementers on aspects of stress in the workplace and interactions with the food environment.

A full table of all measures to be administered is detailed in table 1.

\section{How Science Will Inform Policy}

Promoting health and wellbeing in the workplace is of public health concern, since obesity perpetuates social and health inequalities. It is estimated that $60 \%$ of the UK population could be obese by 2050 [33]. At a national level, the Scottish Government have identified the workplace as a key area for development of 'Healthy Weight Workforce' within the framework of 'Environment \& Health'. In this context, the workplace can be considered a captive microenvironment where health-promoting initiatives can be assessed in terms of behavioural outcomes (eating and physical activity) [34]. Specifically, this could involve education and practical changes in the workplace (e.g. catering provision) to assess impact on body weight and other health indicators (e.g. blood pressure). The planned NeuroFAST studies, in particular, will increase our understanding of stress-induced eating and impact of shift work on eating behaviour in public sector workplaces. This will help inform policy initiatives to reduce stress in the workplace, and shape the environment to reduce the incidence of obesogenic behaviours. In particular, this work will help elucidate how typical stress situations in 'modern life' trigger disadvantageous eating patterns and preference for certain foods (e.g. high-fat or sugar foods). We are trying to understand the underlying physiology and psychology influencing appetite and linking this to eating behaviour. We are particularly interested in factors that influence appetite, since this will impact on longerterm body weight control. However, developing understanding of how to promote health enhancing behaviour(s) is challenging because eating is influenced by a number of internal (within the body and mind) and external (environmental) factors. For example, we do not always initiate eating just when we are hungry; emotions and learned behaviours to 'relieve stress' can contribute to overconsumption of calories.

\section{Policy Relevance - Summary Points}

To elucidate psychological and physiological components of how typical stress situations in 'modern life' trigger disadvantageous eating patterns and preference for certain foods.

To investigate 'stress-induced eating' in the workplace and its potential impact on body weight regulation, with special emphasis on the role of shift work.

To make recommendations on how to shape the workplace food environment to reduce the incidence of disadvantageous eating patterns than can contribute to weight gain and obesity. 
Table 1. Measures to be administered to all participants, in the order of presentation

Description of measure

Initial Visit

Anthropometric measurement

Three Factor Eating inventory (TFEI)

Dutch Eating Behaviour Questionnaire (DEBQ) Qu

Job Content Questionnaire (JCQ)

Food Frequency Questionnaire (FFQ)

Baecke Activity Questionnaire
BMI, waist-hip-ratio, visceral fat percentage

Questionnaire based instrument assessing three domains of eating behaviour; restraint, hunger and disinhibition

\section{of eating behaviour; restraint, emotional and external}

Questionnaire based instrument assessing demand/control and effort/reward aspects of job stress

Self administered booklet asking respondent to report frequency of consumption and portion size of over 120 common foods

Questionnaire for evaluating a person's physical activity and separating it into three distinct dimensions; work, sport and leisure
Interim 7 days

Daily 7-day weighed intake food diary

Hourly VAS for Hunger (during waking hours)

Post MEAL and Snack VAS

Daily Hassles Scale (daily)

End of Day Questionnaire (daily)
Self reported intake of all food and beverages, including time of eating

Self reported visual analogue scale rating of appetite

Self reported visual analogue scale rating of pleasantness and palatability of food

Self report in 7-day diary each stressor and its intensity on 4 point scale; stressors are then coded into egothreatening, interpersonal, work-related or physical

Assesses workload, sleep, mood state (fatigue, anxiety, depression) and motivation to eat (perceived hunger, appetite, food preference)

\section{Final Visit}

Depression, Anxiety and Stress Scales

(DASS-21)

International Personality Item Pool (IPIP)

Multi Perfectionism Scale (MPS)

Emotion Regulation Questionnaire (ERQ)
Assesses three negative emotional states: depression, anxiety and stress

Assesses big five personality traits; openness, conscientousness, extraversion, agreeableness, neuroticism; subjects rate statements on a 5-point Likert-scale in each domain

Questionnaire based instrument assessing three domains of perfectionism; statements are rated on a 5 point scale

Assesses individual differences in the habitual use of two emotion regulation strategies: cognitive reappraisal and expressive suppression 


\section{Acknowledgements}

This research has been funded by the European Union Seventh Framework Programme (FP7/20072013) under grant agreement $n^{\circ} 245009$ and the institute is supported by the Scottish Government Rural and Environment Research and Analysis Directorate (RERAD).

\section{Disclosure Statement}

The authors have no conflicts of interest to report.

\section{References}

1 The World Health Organisation: The World Health Report; Reducing risks, promoting healthy life. WHO, Geneva, 2002.

2 Rogers PJ: Joint Symposium with the British Dietetic Association on 'Implementing Dietary Change; Theory and Practice' Session 3. Changing eating habits. Eating habits and appetite control: a psychobiological perspective. Proc Nutr Soc 1999;58:59-67.

3 Raspopow K, Abizaid A, Matheson K, Anisman H: Psychosocial stressor effects on cortisol and ghrelin in emotional and non-emotional eaters: influence of anger and shame. Horm Behav 2010;58:677-684.

4 Dallman MF: Stress-induced obesity and the emotional nervous system. Trends Endocrinol Metab 2010;21: 159-165.

5 Newman E, O'Connor DB, Conner M: Daily hassles and eating behaviour: The role of cortisol reactivity status. Psychoneuroendocrinology 2007;32:125-132.

6 Wardle J, Steptoe A, Oliver G, Lipsey Z: Stress, dietary restraint and food intake. J Psychosom Res 2000;48: 195-202.

7 O'Connor DB, Jones F, Conner M, McMillan B, Ferguson E: Effects of daily hassles and eating style on eating behavior. Health Psychol 2008;27:S20-S31.

8 Zellner DA, Loaiza S, Gonzalez Z, Pita J, Morales J, Pecora D, Wolf A: Food selection changes under stress. Physiol Behav 2006;87:789-793.

- 9 Overgaard D, Gyntelberg F, Heitman BL: Psychological workload and body weight: Is there an association? A review of the literature. Occup Med 2004;54:35-41.

10 Mind: Stress and Mental Health in the Workplace. Mind Week Report, May 2005. www.mind.org.uk/ assets/0000/6976/Stress_and_MH_in_the_workplace.pdf(last accessed April 3, 3012)..

11 Bradley C, Fisher S, Reason J: Stress and Diabetes; Handbook of Life Stress, Cognition and Health. Oxford, Wiley \& Sons, 1988.

12 Schulte P, Wagner GR, Ostry A, Blanciforti LA, Cutlip RG, Krajnak KM, Luster M, Munson AE, O'Callaghan JP, Parks CG, Simeonova PP, Miller CB: Work, Obesity and Occupational Safety and Health. American J Public Health 2007;97:428-436.

13 Karasek R: Job strain and the prevalence and outcome of coronary artery disease. Circulation 1996;94: 1140-1141.

14 Harrington JM: Health effects of shift work and extended hours of work. Occup Environ Med 2001;58: 68-72.

-15 Lowden A, Moreno C, Holmbäck U, Lennernäs M, Tucker P: Eating and shift work - effects on habits, metabolism, and performance. Scand J Work Environ Health 2010;36:150-162.

16 Wallis DJ, Hetherington MM: Emotions and eating. Self-reported and experimentally induced changes in food intake under stress. Appetite 2009;52:355-362.

17 Dallman MF, Pecoraro NC, La Fleur SE: Chronic stress and comfort foods: self-medication and abdominal obesity. Brain Behav Immun 2005;19:275-280.

18 Torres SJ, Nowson CA: Relationship between stress, eating behavior, and obesity. Nutrition 2007;23:887894.

19 Oliver KG, Huon GF, Zadro L, Williams KD: The role of interpersonal stress in overeating among high and low disinhibitors. Eating Behav 2001;2:19-26.

20 Heatherton TF, Herman CP, Polivy J: Effects of physical threat and ego threat on eating behavior. J Pers Soc Psychol 1991;60:138-143.

-21 Bento C, Pereira AT, Maia B, Marques M, Soares MJ, Bos S, Valente J, Gomes A, Azevedo MHP, Macedo A: Perfectionism and eating behaviour in Portuguese adolescents. Eur Eating Disord Rev 2010;18:328-337.

-22 Bardone-Cone AM, Wonderlich SA, Frost RO, Bulik CM, Mitchell JE, Uppala S, Simonich H: Perfectionism and eating disorders: current status and future directions. Clin Psychol Rev 2007;27:384-405. 
23 Jones CJ, Harris G, Leung N, Blissett J, Meyer C: The effect of induced stress on the relationship between perfectionism and unhealthy eating attitudes. Eating Weight Disord 2007;12:e39-e43.

24 Podar I, Hannus A, Allik J: Personality and affectivity characteristics associated with eating disorders: a comparison of eating disordered, weight-preoccupied, and normal samples. J Pers Assess 1999;73:133-147.

25 Heaven PCL, Mulligan K, Merrilees R, Woods T, Fairooz Y: Neuroticism and conscientiousness as predictors of emotional, external, and restrained eating behaviors. Int J Eating Disord 2001;30:161-166.

-26 O'Connor DB, O'Connor RC: Perceived changes in food intake in response to stress: the role of conscientiousness. Stress Health 2004;20:279-291.

27 Allison DB, Heshka S: Emotion and eating in obesity? A critical analysis. Int J Eating Disord 1993;13:289295.

28 Kaplan HI, Kaplan HS: The psychosomatic concept of obesity. JJ Nerv Ment Dis 1957;125:181-201.

-29 Geliebter A, Aversa A: Emotional eating in overweight, normal weight, and underweight individuals. Eating Behav 2003;3:341-347.

-30 Waters A, Hill A, Waller G: Bulimics' responses to food cravings: is binge-eating a product of hunger or emotional state? Behav Res Ther 2001;39:877-886.

31 Smith A, Johal SS, Wadsworth E, Davey Smith G, Peters T: Scale of Occupational Stress: the Bristol Stress and Health at Work Study. HSE Contract Research Report 265/2000, 2000.

32 Blaug R, Kenyon A, Lekhi R: Stress at Work: A Report Prepared for the Work Foundation's Principal partners, London, 2007.www.theworkfoundation.com/assets/docs/publications/69_stress_at_work.pdf(last accessed April 3, 2012).

33 FORESIGHT: Tackling Obesities: Future Choices - Project Report, 2nd ed. 2007. www.bis.gov.uk/assets/foresight/docs/obesity/17.pdf (last accessed April 23, 2012).

34 The Scottish Government: Healthy Eating, Active Living: An Action Plan to Improve Diet, Increase Physical Activity and Tackle Obesity (2008-2011). 2008. www.scotland.gov.uk/Resource/Doc/228860/0061963.pdf (last accessed April 23, 2012). 